\title{
Nipple-sparing mastectomies: Clinical outcomes from a single academic institution
}

\author{
BARBARA DULL ${ }^{1}$, LEAH CONANT $^{1}$, TERENCE MYCKATYN $^{2}$, \\ MARISSA TENENBAUM ${ }^{2}$, AMY CYR ${ }^{1}$ and JULIE A. MARGENTHALER ${ }^{1}$ \\ ${ }^{1}$ Department of Surgery, Washington University School of Medicine; \\ ${ }^{2}$ Division of Plastic and Reconstructive Surgery, Washington University, St. Louis, MO 63110, USA
}

Received October 11, 2016; Accepted February 15, 2017

DOI: $10.3892 /$ mco.2017.1208

\begin{abstract}
Nipple-sparing mastectomies (NSMs) are increasingly used in the surgical treatment of patients with breast cancer and for prevention of breast cancer. The present study was performed to review the outcomes of patients undergoing NSMs at a single large university setting. A retrospective chart review was performed on all patients undergoing NSMs from 2008-2014. Charts were reviewed for demographic data and patient characteristics. Tumor and breast size, cancer recurrence and complications were also evaluated. Descriptive statistics were utilized to summarize the findings. From 2008-2014, 110 patients underwent 197 NSMs. The mean patient age was 44.4 years (range, 20-77). The average body mass index was 24 (range, 18-47). Breast weight was available for 106 specimens, with a mean weight of $475.5 \mathrm{~g}$ (range, 124.1-1,625.0 g). Seventy-three NSMs were performed for cancer and 124 were performed prophylactically. The mean tumor width was $1.38 \mathrm{~cm}$ (range, $0-6.0 \mathrm{~cm}$ ), with an average nipple to tumor distance of $5.87 \mathrm{~cm}$ (range, 2.93-10.0 cm). Three (4\%) patients required removal of the nipple areolar complex (NAC) due to pathological extension of the tumor. A total of 34 (17.2\%) complications occurred, including infections, hematomas and nipple necrosis, with 9 requiring removal of the NAC and 13 requiring removal of the tissue expander or implant. Smokers had a $36.0 \%$ (9/25) complication rate, compared with $14.5 \%$ (25/172) of nonsmokers $(\mathrm{P}<0.05)$. During follow-up, one recurrence was noted, located on the chest wall. There were no recurrences in the NAC group. Therefore, NSMs may safely be performed without compromising oncologic outcomes or increasing complication rates in properly selected patients.
\end{abstract}

Correspondence to: Dr Julie A. Margenthaler, Department of Surgery, Washington University School of Medicine, CB 8109, 660 S. Euclid Avenue, St. Louis, MO 63110, USA

E-mail: margenthalerj@wudosis.wustl.edu

Key words: nipple-sparing mastectomy, breast cancer, reconstruction

\section{Introduction}

For nearly a century the standard surgical approach to treating breast cancer was to perform a Halstead radical mastectomy removing the breast, axillary lymph nodes and pectoralis muscle. It was believed that the more tissue that was removed the better the survival for the patient. This theory was disproven and surgeons began performing less invasive surgery in the 1970s and 1980s (1,2). With the publication of National Surgical Adjuvant Breast and Bowel Project protocol B-06, breast conserving surgery was determined to have equivalent results in terms of survival and local recurrence with the addition of radiation therapy, when compared with mastectomy (3). If deemed to be an appropriate candidate, patients have the choice between breast conserving surgery or mastectomy. The rates of breast conserving surgery have remained stable over the last decade, however the rates of women opting for contralateral prophylactic mastectomy have steadily increased for various reasons (4). For patients undergoing mastectomies, rates of reconstruction vary and $\leq 42 \%$ undergo this procedure (5). Traditionally, mastectomy with reconstruction for the treatment of breast cancer has been performed via a skin-sparing approach, as first described by Toth and Lappert (6) in 1991. Nipple and skin-sparing mastectomies, with preservation of the nipple-areola complex (NAC), have generally been performed for the treatment of benign disease and prophylactically in women at high risk for the development of breast cancer (7-9).

One of the concerns regarding the safety of nipple-sparing mastectomies (NSMs) in the treatment of breast cancer patients is the potential increased risk of breast cancer recurrence. The terminal duct lobular unit has been identified as the location for the development of all breast cancer, and a 2008 study identified the presence of terminal duct lobular units in the nipples of $9 \%$ of patients $(10,11)$. The rate of occult malignancy detected in the NAC in mastectomy specimens has varied widely with studies reporting an incidence of $1-31 \%$ (12-16). Over the years, a number of prospective and retrospective studies have demonstrated a cancer recurrence rate in the $\mathrm{NAC}$ of up to $2 \%$, with local recurrence rates ranging from 1-6\% (17-19). A more recent meta-analysis comparing NSM to skin-sparing mastectomy (SSM) and/or modified radical mastectomy (MRM) identified no difference 
in overall survival, disease free survival or local recurrence amongst the groups in properly selected patients with early stage breast cancer (20).

Overall complication rates for NSM have been reported to be as high as $60 \%$ in certain older studies, with more recent data suggesting complication rates of $16-22 \%$ (21-22). In a 2003 study published by Gerber et al (19) the complication rate of NSM was compared with SSM and MRM and the overall complication rates were 20,20 , and $18 \%$ respectively. Similar findings have been reported in more recent retrospective studies comparing NSM with SSM $(16,21)$. With comparable complication and recurrence rates to SSM, more surgeons are offering NSM to patients as a more cosmetically appealing alternative.

The current study was undertaken to evaluate the outcomes of patients undergoing NSMs at a single, large university hospital. Specifically, complication rates and tumor recurrence were studied.

\section{Patients and methods}

Patients. A prospectively maintained database of all surgical patients with breast cancer treated between September 2008 and June 2014 was queried following approval from the Washington University Institutional Review Board. A retrospective chart review was then undertaken for all patients who had undergone an NSM performed by one of two breast surgical oncologists with reconstruction performed by one of two plastic surgeons. Charts were reviewed for patient demographics, including age, body mass index (BMI) and smoking status. In those patients who underwent NSMs due to cancer, tumor characteristics were also recorded, including tumor size and nipple to tumor distance as well as tumor recurrence. All patients were required to have a tumor to nipple distance of $>2 \mathrm{~cm}$ to be eligible for nipple-sparing mastectomy at our institution. The incision utilized, as well as the type of reconstruction undertaken were also evaluated. For all patients, any complications occurring within 30 days of surgery were documented, including any additional surgical procedures that were performed.

Statistical analysis. Statistical analysis was performed utilizing Fisher's exact test for categorical variables and student's unpaired $t$-test for continuous variables utilizing SPSS software version 22 (IBM SPSS, Armonk, NY, USA). $\mathrm{P}<0.05$ was considered to indicate a statistically significant difference.

\section{Results}

Patient demographics. In the 6-year study period, 110 patients underwent 197 NSMs. Twenty-three (20.9\%) patients had a unilateral NSM, while 87 (79.1\%) went on to have bilateral NSMs. Of the 197 procedures performed, 124 (62.9\%) were prophylactic and $73(37.1 \%)$ were completed for cancer. Of the patients presenting with a breast cancer diagnosis, 25 (34.2\%) had ductal carcinoma in situ (DCIS), 32 (43.8\%) were TNM Stage I, 14 (19.2\%) were TNM Stage II and 2 (2.8\%) were TNM Stage III. Nine (8\%) patients underwent neoadjuvant chemotherapy, 17 (15.5\%) were treated with adjuvant chemotherapy and $3(2.7 \%)$ underwent neoadjuvant and adjuvant chemotherapy. Two (1.8\%) patients had undergone previous breast conserving therapy with radiation therapy. Ten (9\%) patients underwent adjuvant chest wall radiation due to positive or close $(<1 \mathrm{~mm})$ margins or lymph node involvement on final pathology results.

The mean age of all patients undergoing surgery was 44.4 years (range, 20-77). Overall, the patients in the current study were at a healthy weight with an average BMI of 24, with a BMI range of 18-47. Breast weight was only documented for 106 specimens, with a mean weight of $475.5 \mathrm{~g}$ (range, $124.1-1,625.0 \mathrm{~g})$. Thirteen (11.8\%) of the patients studied were active smokers at the time of surgery.

Complications. The overall complication rate in this study was $17.3 \%$ (34/197; Table I). There was no statistically significant difference in complication rates based on patient age, BMI or breast weight. However, those patients who had complications were more likely to be smokers (26.5\%) compared to patients without any complications $(8.6 \% ; \mathrm{P}<0.05)$. Four of the patients who were smokers developed bilateral complications of infection and nipple necrosis. Thirteen patients underwent removal of their implant or tissue expander due to a complication and this was more common in smokers (25.0\%) than nonsmokers $(4.6 \% ; \mathrm{P}<0.05)$.

There was no difference in the rate of complications, whether the operation was performed prophylactically or as part of breast cancer treatment (Table I). We considered a positive nipple margin to be a complication; therefore those patients with a closer tumor to nipple distance on final pathology had a significantly higher complication rate (Table I). Of the patients with complications, 5 (14.7\%) had undergone either prior chest wall radiation or adjuvant radiation compared with only $7(4.3 \%)$ patients without complications $(\mathrm{P}<0.05)$. The most common complications were skin flap or nipple necrosis (12/34) and infection (11/34) (Table II). Five occurrences of necrosis required NAC reconstruction, 3 instances were extensive necrosis necessitating implant removal and an additional 2 required surgical revision of the incision. Of the 11 infections occurring in the 30-day postoperative period, 9 required removal of the implant and 1 patient required surgical revision of their wound. Five hematomas occurred postoperatively with 3 requiring surgery for evacuation of the hematoma and 1 necessitating implant removal. A single seroma occurred in the 30-day postoperative period, which was treated with needle aspiration. One patient developed flash pulmonary edema in the immediate postoperative period but had had bilateral NSMs and therefore was considered to have had two complications.

Surgical technique. Twenty-seven (13.7\%) NSMs were completed via a circumareolar approach, 71 (36\%) via a lateral breast incision and 99 (50.3\%) utilizing an inframammary incision. There was no difference in overall complication rates among the three incision types utilized (Table I). However, when evaluating the 9 complications that resulted in loss of the NAC, there was a significant difference amongst the 3 incision types utilized (Table III). Seven of the 9 (77.8\%) instances of NAC loss occurred following the use of a circumareolar incision compared to 1 (11.1\%) loss each with the lateral and inframammary approaches $(\mathrm{P}<0.05)$. The removal of the 
Table I. Comparison of demographics, tumor characteristics and surgical data of patients undergoing nipple-sparing mastectomies with and without subsequent complications.

\begin{tabular}{|c|c|c|c|}
\hline & No complications $(n=163)$ & Complications $(n=34)$ & P-value \\
\hline Patient age, years (range) & $44.1(25-77)$ & $43.6(20-72)$ & NS \\
\hline BMI (range) & $25.2(19.9-31.2)$ & $23.7(17.9-47.2)$ & NS \\
\hline Smoker $(\%)$ & $14(8.6)$ & $9(26.5)$ & $<0.01$ \\
\hline Prophylactic surgery (\%) & $105(64.4)$ & $19(55.8)$ & NS \\
\hline \multicolumn{4}{|l|}{ Stage $(\%)$} \\
\hline 0 & $21(12.9)$ & $4(11.8)$ & NS \\
\hline $\mathrm{I}$ & $26(16)$ & $6(17.6)$ & NS \\
\hline II & $10(6.1)$ & $4(11.8)$ & NS \\
\hline III & $1(0.6)$ & $1(3)$ & NS \\
\hline Breast weight, $\mathrm{g}$ (range) ${ }^{\mathrm{a}}$ & $458(124-1625)$ & $545(170-836)$ & NS \\
\hline Tumor size, $\mathrm{cm}(\text { range })^{\mathrm{b}}$ & $1.5(0-6)$ & $1.0(0-1.05)$ & NS \\
\hline Tumor to nipple distance, $\mathrm{cm}(\text { range })^{\mathrm{c}}$ & $5.87(2.93-10.7)$ & $5.23(0-8.51)$ & $<0.05$ \\
\hline Chest wall radiation & $7(4.3)$ & $5(14.7)$ & $<0.05$ \\
\hline \multicolumn{4}{|l|}{ Incision utilized } \\
\hline Circumareolar & $19(11.7)$ & $8(23.5)$ & NS \\
\hline Lateral & $62(38.0)$ & $9(26.5)$ & NS \\
\hline Inframammary & $82(50.3)$ & $17(50)$ & NS \\
\hline \multicolumn{4}{|l|}{ Reconstruction } \\
\hline Tissue expander & $18(52.9)$ & $88(54)$ & NS \\
\hline Direct to implant & $16(47.1)$ & $75(46)$ & NS \\
\hline
\end{tabular}

Table II. All complications $(\mathrm{n}=34)$ and additional surgical procedures performed to correct the associated complication.

\begin{tabular}{lcl}
\hline Complication type & Number of complications & Additional procedures required (n) \\
\hline Positive nipple margin & 3 & Removal of NAC (3) \\
Hematoma & 5 & Evacuation (3), implant removal (1) \\
Infection & 11 & Implant removal (9), wound revision (1) \\
Necrosis & 12 & NAC reconstruction (5), implant removal (3), \\
& & revision of incision (2) \\
Seroma & 1 & Aspiration \\
Flash pulmonary edema & 2 & None \\
\hline
\end{tabular}

NAC, nipple areolar complex.

NAC that occurred after both a lateral and inframammary approach was due to DCIS behind the nipple, whereas only 1 NAC was removed following a circumareolar incision due to a positive nipple margin. The type of reconstruction performed did not affect complication rates (Table I). Overall, $106(53.8 \%)$ patients underwent reconstruction with tissue expander placement and $91(46.2 \%)$ received direct-to-implant based reconstruction. There was no difference in complication rate among patients receiving a tissue expander $(16.9 \%)$ and direct implant (17.6\%) $(\mathrm{P}>0.05)$. However, a significantly larger percentage of patients receiving a direct implant (10.9\%) underwent implant removal due to a complication than those with placement of a tissue expander $(2.8 \% ; \mathrm{P}<0.05)$.

Outcomes. In the first 3 years of the study, 44 NSMs were performed with $9(20.5 \%)$ complications, compared with a complication rate of $16.3 \%(25 / 153)$ in the final 3 years of the study $(\mathrm{P}>0.05)$. The complication rate has steadily declined, with only $5 / 37(13.5 \%)$ patients experiencing a complication occurring in the most recent six months of the study. 
Table III. Comparison of the incision utilized and the rate of NAC loss.

\begin{tabular}{lcc}
\hline Incision utilized & Loss of NAC (n=9) & P-value \\
\hline Circumareolar, n (\%) & $7(77.8)$ & $<0.05$ \\
Lateral, n (\%) & $1(11.1)$ & \\
Inframammary, n (\%) & $1(11.1)$ & \\
\hline
\end{tabular}

P-value calculated using Fisher's exact test. NAC, nipple areolar complex.

Follow-up ranged between 3 months and 6 years, and only one (1.4\%) patient in the cohort who underwent an NSM for cancer ultimately developed a recurrence. This occurred in a patient with a T1N0 tumor, $4.75 \mathrm{~cm}$ from the NAC who developed a chest wall recurrence. There were no instances of tumor recurrence within the NAC in the current study.

\section{Discussion}

The rate of local recurrence in numerous studies of patients undergoing an NSM varies, ranging from $0-25.7 \%(18,19,23,24)$. In the studies demonstrating rates of local recurrence $>10 \%$, cancer recurrence in the lymph nodes was included in the rate $(19,24)$. Gerber et al (19) evaluated 246 patients with an indication for MRM who were treated with MRM, SSM or NSM with axillary lymph node dissection, identifying no differences in the rates of local recurrence amongst the three groups after a mean follow-up period of 101 months. More recent studies have demonstrated much lower local recurrence rates, similar to the present findings $(16,24)$. An analysis of 1,006 procedures from the Italian National database of NSM, the local regional recurrence rate was $2.9 \%$ with a recurrence rate in the NAC of $0.7 \%$ (25). In the present study, a single recurrence occurred, yielding a local recurrence rate of $1.4 \%$. This was a chest wall recurrence in a patient with stage I (T1NO) cancer who did not require adjuvant chemotherapy or radiation following NSM. No patients presented with recurrence in the NAC during the time period studied.

In order to decrease the likelihood of cancer involvement of the NAC, numerous studies have recommended that the tumor to NAC distance on pre-operative imaging be no closer than $2 \mathrm{~cm}(23,26-28)$. Tumors found to be closer than $2 \mathrm{~cm}$ to the NAC are considered to be a relative contraindication to pursuing NSM. The lower local recurrence rate in the current study is likely due in large part to patient selection, as there was a requirement that all imaging demonstrate a distance $>2 \mathrm{~cm}$ between the tumor and the NAC. A previous study by Tang et al (29) evaluated their group's experience with positive nipple margins and management, including excision of only the nipple with retention of the areola, and identified that at a 36-month median follow-up, no recurrences occurred in the nipple/NAC. This may expand the indications for NSM in the future.

The complication rate in the present study was $17.3 \%$, which is comparable with the reported literature $(21,25,30,31)$. In the current study, those patients with a closer tumor to nipple distance had a higher complication rate. Any patient requiring excision of the nipple-areolar complex (NAC) for any reason was considered to have had a complication. Therefore a higher complication rate associated with a close tumor to nipple distance is attributed to the three patients who had excision of the NAC for a final positive nipple margin with involvement of DCIS. The closest tumor to nipple margin, without a positive margin or complication, in this study was found to be $2.93 \mathrm{~cm}$.

Although the rate of overall complications was similar from 2008-2011 and 2012-2014, when examining the data for 2014, as experience with the operation has increased the complication rate has decreased to $13.5 \%$. As this is becoming a more common option for patients, the present study anticipates a further decline in complications.

Smoking is established to increase complication rates in breast surgery $(5,31-36)$. In a prospective study published by Matsen et al (33) examining skin flap necrosis rates following any mastectomy with reconstruction, $14 \%$ of patients had some level of skin flap necrosis (33). Using univariate analysis, smoking was revealed to be a significant factor in necrosis rates (32). Similarly, in a study published in $2014,6 \%$ of patients undergoing NSM were found to be smokers, and had an odds ratio of 3.3 for any complications compared with non-smokers (31). This trend was also evident in the present study, with patients who were smokers at the time of NSM having significantly more complications than non-smokers and requiring removal of their implants or tissue expanders due to these complications.

One of the most well-described complications to occur after an NSM is skin or nipple necrosis, which at times necessitates removal of the NAC. In the majority of contemporary studies, the necrosis rate has been found to be between 0 and $7 \%(21,22,25,31)$. In the current study the total necrosis rate was $6 \%$, with $5(2.5 \%)$ patients requiring removal and reconstruction of the NAC due to the extent of necrosis.

When NSMs were first being performed at the Washington University School of Medicine, one of three incision types was utilized: Circumareolar (which encompassed a portion of the NAC border), lateral radial and inframammary. The circumareolar approach was replaced by the lateral radial or inframammary incisions, as there was significantly less loss of the NAC when the incision did not involve the NAC. This has been demonstrated by numerous other studies $(28,34,37)$. In a 2014 retrospective review of 500 NSMs, patients with incisions encompassing the NAC had a complication rate of $21.1 \%$ compared to $8.5 \%$ when the inframammary incision was used (31). A meta-analysis of 48 studies published between 1970 and 2013 evaluated whether incision type led to an increase in nipple necrosis, and revealed that a circumareolar incision yielded a $17.81 \%$ necrosis rate, whereas while radial/ lateral and inframammary incisions had necrosis rates of 8.83 and $9.09 \%$, respectively (21). Due to the increased number of complications and rate of nipple loss with a circumareolar approach in both the literature and in the present study, this has been abandoned at Washington University School of Medicine and NSMs are now performed through a lateral or inframammary incision only.

Historically, patients who underwent implant-based reconstruction following NSM had tissue expanders placed at the 
time of mastectomy, however immediate reconstruction with a silicone or saline implant following NSM has become more widely accepted in recent years. In a 2010 prospective study of 214 consecutive NSMs with immediate silicone implant reconstruction, the overall complication rate was $16 \%$ with $6 \%$ requiring explantation of the prostheses due to the complication (22). In 2012 the present study began performing NSM with direct-to-implant reconstruction and in the study period identified no difference in complication rates when a permanent implant was placed at the time of mastectomy in lieu of a tissue expander; however, more patients with direct implant-based reconstruction required removal of their implants.

There are limitations to the present study, as it was a retrospective chart review and certain medical data was missing or incomplete. For example, breast weight was only recorded for 106 breast specimens and therefore the current study was unable to delineate whether larger breast size increases complication rates. Although this is a retrospective study, it provides additional data regarding the oncologic safety and low complication rate of NSMs. It is unlikely that a randomized controlled trial may be offered to patients comparing NSM to SSM and MRM, as patients who are candidates for a NSM would not want to be randomized to a less cosmetically pleasing surgery.

In conclusion, the present study adds to the growing literature demonstrating the oncologic safety of NSMs with a local recurrence rate of $1.4 \%$ in properly selected patients with tumor to nipple distance $>2 \mathrm{~cm}$. Additionally, the current study also demonstrated a low complication rate, including a low rate of nipple-areolar loss with a lateral or inframammary incision. These results further demonstrate that NSMs are safe and do not comprise oncologic outcomes or increase complication rates.

\section{References}

1. Fisher B, Montague E, Redmond C, Barton B, Borland D Fisher ER, Deutsch M, Schwarz G, Margolese R, Donegan W, et al: Comparison of radical mastectomy with alternative treatments for primary breast cancer. A first report of results from a prospective randomized clinical trial. Cancer 39: S2827-S2839, 1977.

2. Fisher B, Montague E, Redmond C, Deutsch M, Brown GR, Zauber A, Hanson WF and Wong A: Findings from NSABP Protocol No. B-04-comparison of radical mastectomy with alternative treatments for primary breast cancer. I. Radiation compliance and its relation to treatment outcome. Cancer 46: $1-13,1980$.

3. Fisher B, Bauer M, Margolese R, Poisson R, Pilch Y, Redmond C, Fisher E, Wolmark N, Deutsch M, Montague E, et al: Five-year results of a randomized clinical trial comparing total mastectomy and segmental mastectomy with or without radiation in the treatment of breast cancer. N Engl J Med 312: 665-673, 1985.

4. Tuttle TM, Rueth NM, Abbott A and Virnig BA: Trends in the local treatment of breast cancer: Should we be worried? J Surg Oncol 103: 313-316, 2011.

5. Morrow M, Li Y, Alderman AK, Jagsi R, Hamilton AS, Graff JJ, Hawley ST and Katz SJ: Access to breast reconstruction after mastectomy and patient perspectives on reconstruction decision making. JAMA Surg 149: 1015-1021, 2014.

6. Toth BA and Lappert P: Modified skin incisions for mastectomy: The need for plastic surgical input in preoperative planning. Plast Reconstr Surg 87: 1048-1053, 1991.

7. Freeman BS: Subcutaneous mastectomy for benign breast lesions with immediate or delayed prosthetic replacement. Plast Reconstr Surg Transplant Bull 30: 676-682, 1962.

8. Hartmann LC, Schaid DJ, Woods JE, Crotty TP, Myers JL, Arnold PG, Petty PM, Sellers TA, Johnson JL, McDonnell SK, et al: Efficacy of bilateral prophylactic mastectomy in women with a family history of breast cancer. N Engl J Med 340: 77-84, 1999.
9. Pennisi VR and Capozzi A: Subcutaneous mastectomy data: A final statistical analysis of 1500 patients. Aesthetic Plast Surg 13: 15-21, 1989

10. Wellings SR: A hypothesis of the origin of human breast cancer from the terminal ductal lobular unit. Pathol Res Pract 166: 515-535, 1980.

11. Stolier AJ and Wang J: Terminal duct lobular units are scarce in the nipple: Implications for prophylactic nipple-sparing mastectomy: Terminal duct lobular units in the nipple. Ann Surg Oncol 15: 438-442, 2008.

12. Parry RG, Cochran TC Jr and Wolfort FG: When is there nipple involvement in carcinoma of the breast? Plast Reconstr Surg 59: 535-537, 1977.

13. Morimoto T, Komaki K, Inui K, Umemoto A, Yamamoto $\mathrm{H}$, Harada $\mathrm{K}$ and Inoue $\mathrm{K}$ : Involvement of nipple and areola in early breast cancer. Cancer 55: 2459-2463, 1985.

14. Wang J, Xiao X, Wang J, Iqbal N, Baxter L, Skinner KA, Hicks DG, Hajdu SI and Tang P: Predictors of nipple-areolar complex involvement by breast carcinoma: Histopathologic analysis of 787 consecutive therapeutic mastectomy specimens. Ann Surg Oncol 19: 1174-1180, 2012.

15. Billar JA, Dueck AC, Gray RJ, Wasif $N$ and Pockaj BA: Preoperative predictors of nipple-areola complex involvement for patients undergoing mastectomy for breast cancer. Ann Surg Oncol 18: 3123-3128, 2011.

16. Wijayanayagam A, Kumar AS, Foster RD and Esserman LJ: Optimizing the total skin-sparing mastectomy. Arch Surg 143: 38-45, 2008.

17. Petit JY, Veronesi U, Orecchia R, Curigliano G, Rey PC, Botteri E, Rotmensz N, Lohsiriwat V, Cassilha Kneubil M and Rietjens M: Risk factors associated with recurrence after nipple-sparing mastectomy for invasive and intraepithelial neoplasia. Ann Oncol 23: 2053-2058, 2012.

18. Caruso F, Ferrara M, Castiglione G, Trombetta G, De Meo L, Catanuto G and Carillio G: Nipple sparing subcutaneous mastectomy: Sixty-six months follow-up. Eur J Surg Oncol 32: 937-940, 2006.

19. Gerber B, Krause A, Dieterich M, Kundt G and Reimer T: The oncological safety of skin sparing mastectomy with conservation of the nipple-areola complex and autologous reconstruction: An extended follow-up study. Ann Surg 249: 461-468, 2009.

20. De La Cruz L, Moody AM, Tappy EE, Blankenship SA and Hecht EM: Overall survival, disease-free survival, local recurrence and nipple-areolar recurrence in the setting of nipple-sparing mastectomy: A meta-analysis and systematic review. Ann Surg Oncol 22: 3241-3249, 2015.

21. Endara M, Chen D, Verma K, Nahabedian MY and Spear SL: Breast reconstruction following nipple-sparing mastectomy: A systematic review of the literature with pooled analysis. Plast Reconstr Surg 132: 1043-1054, 2013.

22. Radovanovic Z, Radovanovic D, Golubovic A, Ivkovic-Kapicl T, Bokorov B and Mandic A: Early complications after nipple-sparing mastectomy and immediate breast reconstruction with silicone prosthesis: Results of 214 procedures. Scand J Surg 99: 115-118, 2010.

23. Jensen JA, Orringer JS and Giuliano AE: Nipple-sparing mastectomy in 99 patients with a mean follow-up of 5 years. Ann Surg Oncol 18: 1665-1670, 2011.

24. Benediktsson KP and Perbeck L: Survival in breast cancer after nipple-sparing subcutaneous mastectomy and immediate reconstruction with implants: A prospective trial with 13 years median follow-up in 216 patients. Eur J Surg Oncol 34: 143-148, 2008.

25. Orzalesi L, Casella D, Santi C, Cecconi L, Murgo R, Rinaldi S, Regolo L, Amanti C, Roncella M, Serra M, et al: Nipple sparing mastectomy: Surgical and oncological outcomes from a national multicentric registry with 913 patients (1006 cases) over a six year period. Breast 25: 75-81, 2016.

26. Vlajcic Z, Zic R, Stanec S, Lambasa S, Petrovecki M and Stanec Z: Nipple-areola complex preservation: Predictive factors of neoplastic nipple-areola complex invasion. Ann Plast Surg 55: 240-244, 2005.

27. Pirozzi PR, Rossetti C, Carelli I, Ruiz CA, Pompei LM and Piato S: Clinical and morphological factors predictive of occult involvement of the nipple-areola complex in mastectomy specimens. Eur J Obstet Gynecol Reprod Biol 148: 177-181, 2010.

28. Mallon P, Feron JG, Couturaud B, Fitoussi A, Lemasurier P, Guihard T, Cothier-Savay I and Reyal F: The role of nipple-sparing mastectomy in breast cancer: A comprehensive review of the literature. Plast Reconstr Surg 131: 969-984, 2013. 
29. Tang R, Coopey SB, Merrill AL, Rai U, Specht MC, Gadd MA, Colwell AS, Austen WG Jr, Brachtel EF and Smith BL: Positive nipple margins in nipple-sparing mastectomies: Rates, management and oncologic safety. J Am Coll Surg 222: 1149-1155, 2016.

30. Gould DJ, Hunt KK, Liu J, Kuerer HM, Crosby MA, Babiera G and Kronowitz SJ: Impact of surgical techniques, biomaterials, and patient variables on rate of nipple necrosis after nipple-sparing mastectomy. Plast Reconstr Surg 132: 330e-338e, 2013.

31. Colwell AS, Tessler O, Lin AM, Liao E, Winograd J, Cetrulo CL, Tang R, Smith BL and Austen WG Jr: Breast reconstruction following nipple-sparing mastectomy: Predictors of complications, reconstruction outcomes, and 5-year trends. Plast Reconstr Surg 133: 496-506, 2014

32. Abedi N, Ho AL, Knox A, Tashakkor Y, Omeis T, Van Laeken N, Lennox P and Macadam SA: Predictors of mastectomy flap necrosis in patients undergoing immediate breast reconstruction: A review of 718 patients. Ann Plast Surg 76: 629-634, 2016.

33. Matsen CB, Mehrara B, Eaton A, Capko D, Berg A, Stempel M, Van Zee KJ, Pusic A, King TA, Cody HS III, et al: Skin flap necrosis after mastectomy with reconstruction: A prospective study. Ann Surg Oncol 23: 257-264, 2016.
34. Petersen A, Eftekhari AL and Damsgaard TE: Immediate breast reconstruction: A retrospective study with emphasis on complications and risk factors. J Plast Surg Hand Surg 46: 344-348, 2012.

35. Bertoni DM, Nguyen D, Rochlin D, Hernandez-Boussard T, Meyer S, Choy N, Gurtner GC and Wapnir IL: Protecting nipple perfusion by devascularization and surgical delay in patients at risk for ischemic complications during nipple-sparing mastectomies. Ann Surg Oncol 23: 2665-2672,2016.

36. Chung CU, Wink JD, Nelson JA, Fischer JP, Serletti JM and Kanchwala SK: Surgical site infections after free flap breast reconstruction: An analysis of 2,899 patients from the ACS-NSQIP datasets. J Reconstr Microsurg 31: 434-441, 2015.

37. Garwood ER, Moore D, Ewing C, Hwang ES, Alvarado M, Foster RD and Esserman LJ: Total skin-sparing mastectomy: Complications and local recurrence rates in 2 cohorts of patients. Ann Surg 249: 26-32, 2009. 